\title{
Estimation of Phonon Dispersion Relations Using Correlation Effects Among Thermal Displacements of Atoms
}

\author{
Y. Ishikawa ${ }^{1,2}$, T. Sakuma ${ }^{1 *}$, T. Hashimoto ${ }^{1}$, Xianglian $^{3}$, \\ O. Kamishima ${ }^{4}$ and S.A. Danilkin ${ }^{5}$ \\ ${ }^{1}$ Institute of Applied Beam Science, Ibaraki University, Mito 310-8512, Japan. \\ ${ }^{2}$ Institute of Material Structure Science, KEK Tokai, Shirakata 203-1, Naka 319-1106, Japan. \\ ${ }^{3}$ College of Physics and Electronics Information, Inner Mongolia University for the Nationalities, Tongliao 028043, China. \\ ${ }^{4}$ Institute for Fundamental Sciences, Faculty of Science and Engineering, Setsunan University, Neyagawa 572-8508, Japan. \\ ${ }^{5}$ Bragg Institute, Australian Nuclear Science and Technology Organization, Kirrawee DC NSW 2232, Australia.
}

\section{ARTICLE INFO}

\section{Article history:}

Received 04 October 2014

Received in revised form 22 January 2015

Accepted 30 March 2015

\section{Keywords:}

Neutron diffraction

Diffuse scattering

Correlation effects

Force constants

Phonon

\begin{abstract}
A B S T R A C T
Neutron diffraction measurement of powder $\alpha$-Fe sample at $295 \mathrm{~K}$ was carried out at the high resolution powder diffractometer installed at Japan Proton Accelerator Research Complex (J-PARC). Crystal parameters were determined from Rietveld analysis. The correlation effects among thermal displacements of atoms were estimated from a generalized equation based on the results of fomer diffuse scattering analysis. The force constants among atoms were obtained using an equation for transforming of the correlation effects to force constants. The force constants and the crystal structure of $\alpha-\mathrm{Fe}$ were used to estimate the phonon dispersion relations, phonon density of states, and specific heat by computer simulation. The obtained force constants among first-nearest-neighboring atoms is $2.3 \mathrm{eV} / \AA^{2}$ at $295 \mathrm{~K}$ and the specific heat is $185 \mathrm{meV} / \mathrm{K}$ at $150 \mathrm{~K}$. The calculated phonon dispersion relations and specific heat of $\alpha-\mathrm{Fe}$ are similar to those obtained from inelastic neutron scattering and specific heat measurements, respectively.
\end{abstract}

(C) 2015 Atom Indonesia. All rights reserved

\section{INTRODUCTION}

Diffuse scattering data are very important in the analysis of the static and dynamic disorder in crystals[1]. The static disorder is related to atomic arrangements and atomic defects. For instance, the short-range order in binary alloys has been studied for a long time. The application of diffuse scattering measurements to the problems of dynamic disorder has been started by neutron diffraction measurement. Broad oscillations of the diffuse scattering intensity of ordered crystals, where there are no static disorder, were observed and explained by the correlation effects among thermal displacements of atoms[2-6].

\footnotetext{
* Corresponding author.

E-mail address: sakuma@mx.ibaraki.ac.jp

DOI: http://dx.doi.org/10.17146/aij.2015.349
}

The interatomic force constant among first nearest neighboring atoms had been obtained from the thermal displacements of atoms in the extended X-ray absorption fine structure (EXAFS) analysis [7-9]. Recently, neutron diffraction measurement of an aluminum powder sample was carried out at the high resolution powder diffractometer installed at JRR-3[10]. The interatomic force constants among the first-, second-, and third-nearest-neighboring atoms were determined from the correlation effects using a newly introduced equation of transforming. The derived force constants and the crystal structure of fcc $\mathrm{Al}$ were used to estimate the phonon dispersion relations, phonon density of states, and specific heat by computer simulation.

In this paper, the correlation effects of bcc $\mathrm{Fe}(\alpha-\mathrm{Fe})$ are estimated from interatomic distances using a generalized equation for the former diffuse 
scattering analysis. An algorithm for transforming of the correlation effects among thermal displacements of atoms to force constants is applied to determine the force constants. The force constants of $\alpha-\mathrm{Fe}$ are used to estimate the phonon dispersion relations, phonon density of states, and specific heat by computer simulation. The calculated phonon dispersion relations and specific heat are compared with those obtained by inelastic neutron scattering and specific heat measurements of $\alpha-\mathrm{Fe}[11-13]$.

\section{EXPERIMENTAL METHODS}

The powder $\alpha$-Fe sample $(99.9 \%$, Kojundo Chemical) was sealed in V-Ni can with $6 \mathrm{~mm}$ diameter. Since the small vanadium Bragg peaks will be detected from a high flux beam experiment in J-PARC, the vanadium sample container alloyed with nickel which cancels the vanadium coherent scattering amplitude was used [14].

Neutron diffraction measurements were performed at $295 \mathrm{~K}$ with IBARAKI Materials Design Diffractometer (iMATERIA) installed at the Materials and Life Science Experimental Facility (MLF) in J-PARC [15]. The diffraction intensities were collected by time of flight (TOF) method. The effective $d$-range was from $0.32 \AA$ to $2.8 \AA$ in back scattering bank.

The observed powder diffraction intensities of $\alpha$-Fe at $295 \mathrm{~K}$ are shown in Fig. 1. Calculations by Rietveld analysis were performed using Z-Rietveld [16]. The crystal structure of $\alpha$-Fe in the Rietveld analysis belongs to a bcc type with the space group $\mathrm{Im}-3 \mathrm{~m}$. The iron atoms occupy $2 a$ positions. The refined structural parameters of lattice constant and the parameter of Debye-Waller temperature factor are $a=2.866566(2) \AA$ and $B=0.270(1) \AA^{2}$, respectively. The diffuse scattering intensity of $d$ spacing is shown in Fig. 2. There are oscillatory peaks in the diffuse scattering near $d \sim 0.34,0.39$ and 0.47 .

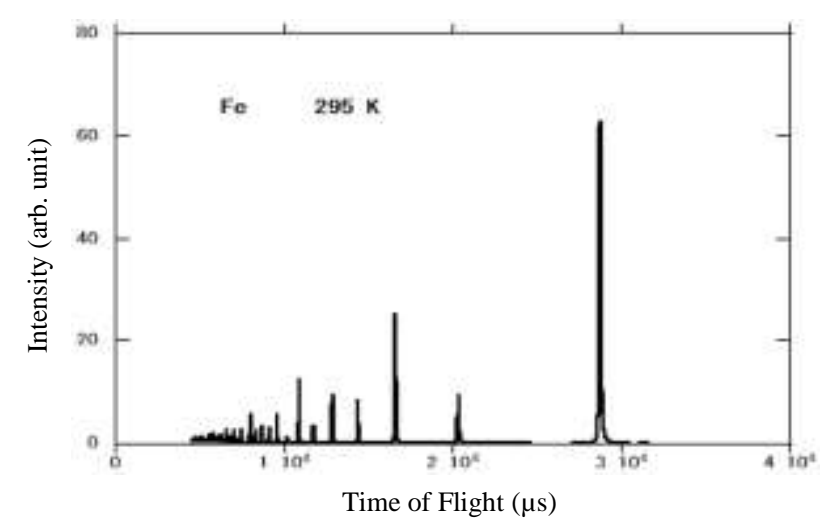

Fig. 1. Powder diffraction pattern of $\alpha-\mathrm{Fe}$ at $295 \mathrm{~K}$.

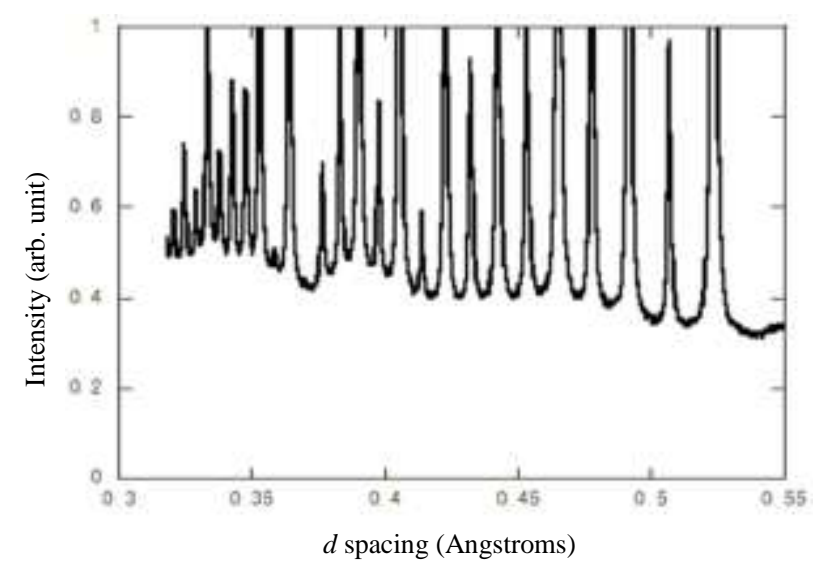

Fig. 2. Diffuse scattering of $\alpha-\mathrm{Fe}$ at $295 \mathrm{~K}$.

\section{RESULTS AND DISCUSSION}

The correlation effects had been determined from the analysis of oscillatory diffuse scattering intensity. The values of correlation effects of fcc metals $(\mathrm{Cu}$ [6], $\mathrm{Al}[10])$ and semiconductor (Ge [2]) near room temperature are shown in Fig. 3. The correlation effects decrease with the increase of interatomic distance. The values of the correlation effects are about 0.6 for the interatomic distance $2.5 \AA$ and about 0.2 for $4 \AA$ near room temperature. The correlation effects do not depend significantly on the type of crystal structure and crystal binding. From the results we suppose an equation between correlation effects $\mu(\gtrsim 0)$ and interatomic distance $r(\AA)$ as

$$
\mu=-0.32 r+1.44
$$

The quantitative analysis of diffuse scattering intensity by TOF diffractometer is difficult. The diffuse scattering intensity includes undefined contributions besides thermal disorder. Although oscillatory diffuse scattering intensity can be observed below $d<0.55$ in Fig. 2, the diffuse scattering intensity is weak in the region of $d>0.55$. The oscillatory diffuse scattering part in Figure 2 is mainly from the thermal disorder. The oscillatory peak positions $(d \sim 0.34, \quad 0.39$ and 0.47) correspond to the calculated values with interatomic distance $\left(r_{1}=2.864\right)$ of first-nearest-neighboring atoms: $\sin \left(2 \pi r_{1} / d\right)=1$ [1]. In this paper we estimate the correlation effects of $\alpha-\mathrm{Fe}$ using Eq. (1) without analyzing observed diffuse scattering intensity. The obtained correlation effects among first-, second-, and third -nearest-neighboring atoms in $\alpha-\mathrm{Fe}$ at $295 \mathrm{~K}$ were $0.65,0.52$, and 0.14 , respectively (Table 1 ). 


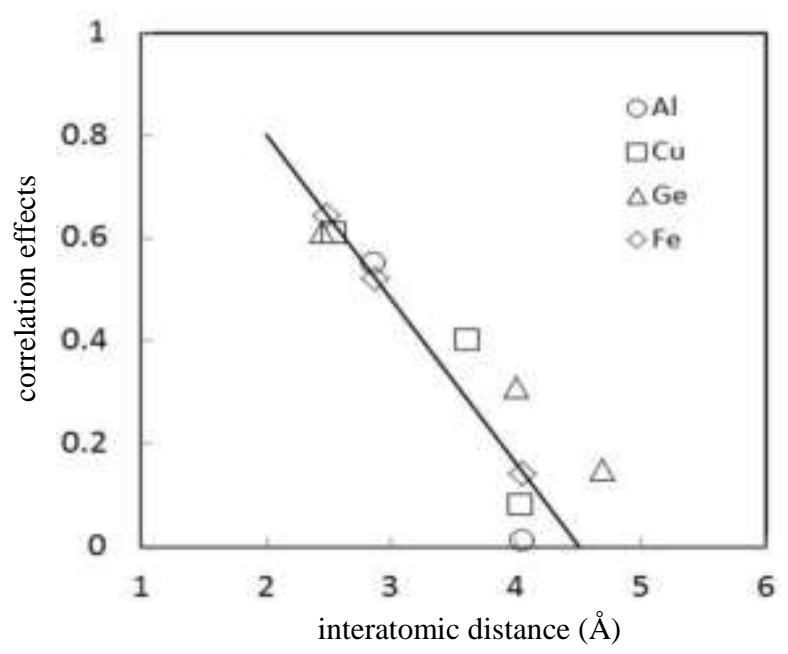

Fig. 3. Correlation effects reported from the analysis of metal and semiconductor at room temperature. A generalized equation (Eq. (1)) is shown by solid line.

Table 1. Interatomic distances $r$, coordination numbers $Z$, correlation effects among thermal displacements of atoms, and force constants of first-, second-, and third-nearest-neighboring atoms of $\alpha-\mathrm{Fe}$ at $295 \mathrm{~K}$.

\begin{tabular}{llll}
\hline & $1 \mathrm{st}$ & 2 nd & 3rd \\
\hline$r(\AA)$ & 2.483 & 2.867 & 4.054 \\
$Z$ & 8 & 6 & 12 \\
$\mu$ & 0.65 & 0.52 & 0.14 \\
$\alpha\left(\mathrm{eV} / \AA^{2}\right)$ & 2.3 & 1.4 & 0.20 \\
\hline
\end{tabular}

An equation for transforming of the correlation effects to force constants has been introduced in the analysis of diffuse scattering of fcc Al [10]. The force constant $\alpha$ between $s$ and $s^{\prime}$ sites is shown as

$$
\alpha=\frac{8 \pi^{2} k_{B} T \mu_{s s^{\prime}}}{3\left(B_{s}+B_{s^{\prime}}\right)\left(1-\mu_{s s^{\prime}}\right)}
$$

where $k_{B}$ is Boltzmann constant and $T$ temperature. In the absence of correlation effects $(\mu=0)$, the force constant is also zero in Eq. (2). The values of correlation effects between the first-, second- and third-nearest- neighboring atoms calculated using Eq. (2) for $\alpha$-Fe at $295 \mathrm{~K}$ are given in Table 1.

We estimate the phonon dispersion relations from the force constants using the method described in Ref.[9]. The calculated phonon dispersion relations propagating in the [100], [110], and [111] directions at $295 \mathrm{~K}$ are shown in Fig. 4. From the phonon dispersion relations, the phonon density of states and specific heat of $\alpha$-Fe were also derived. The results are shown in Figs. 5 and 6. The phonon dispersion relations calculated with force constants determined by Eq. (2) are qualitatively in agreement with the results of inelastic neutron scattering measurements at room temperature [11,12]. For example, there are two transverse acoustic branches $\mathrm{T}_{1}$ and $\mathrm{T}_{2}$ in the dispersion relations in the [110] direction in Fig. 4. A separation into two transverse acoustic branches of [110] direction and the maxima in LA modes of [100] and [111] directions are strongly related to correlation effects of first- and second-nearest-neighboring atoms. The specific heat of Fe below $200 \mathrm{~K}$ is from the contribution of lattice vibrations which corresponds to the calculation of the present phonon dispersion relations. Above $200 \mathrm{~K}$ specific heat related to $\alpha-\gamma$ phase transition is added [13]. The difference between observed ( $\square$ ) and calculated (solid line) values is mainly from the contribution of $\alpha-\gamma$ phase transition..

As the interatomic distance of first-nearestneighboring atoms for $\alpha-\mathrm{Fe}\left(r_{1}=2.483\right)$ is less than that for $\mathrm{Al}\left(r_{1}=2.864\right)$, the correlation effects among first-nearest-neighboring atoms for bcc $\alpha$-Fe $\left(\mu_{1}=0.65\right)$ are greater than that for $\mathrm{fcc} \mathrm{Al}\left(\mu_{1}=0.55\right)$. Young's modulus $E$ of $\alpha-\mathrm{Fe}$ is $15.23 \times 10^{10} \mathrm{~N} / \mathrm{m}^{2}$ and that of Al $7.03 \times 10^{10} \mathrm{~N} / \mathrm{m}^{2}$ [17]. The ratio of force constant among first-nearest- neighboring atoms for $\alpha$-Fe to that of $\mathrm{Al}$ is similar to the ratio of Young's modulus.

The estimation of correlation effects in other metals would be possible from Eq. (1). The force constants are obtained from the correlation effects. By a computer simulation the phonon dispersion relations and specific heat of metals can be estimated.
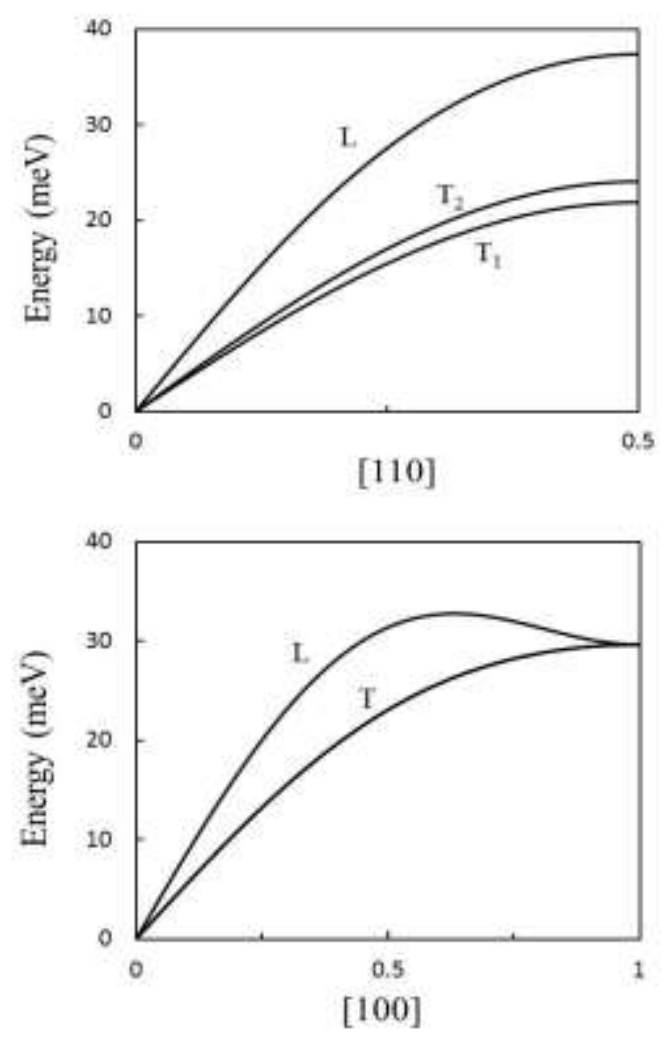


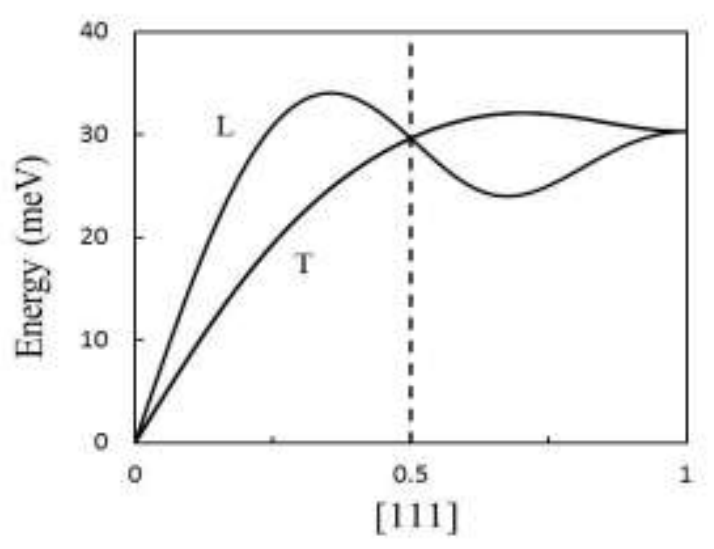

Fig. 4. Phonon dispersion relations of $\alpha-\mathrm{Fe}$ in the [100], [110], and [111] directions at $295 \mathrm{~K}$ calculated using the force constants determined using Eq. (2). The dashed line in [111] direction shows zone boundary.

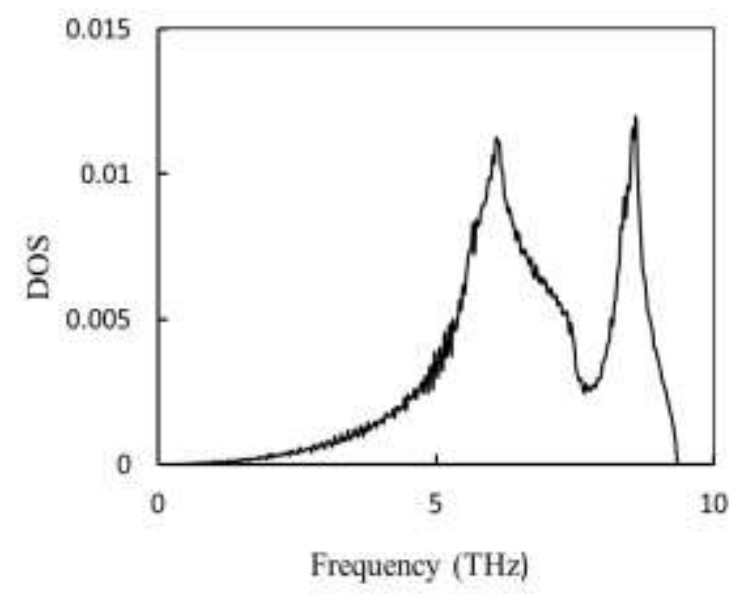

Fig. 5. Phonon density of states of $\alpha$-Fe calculated by computer simulation using the force constants determined using Eq. (2).

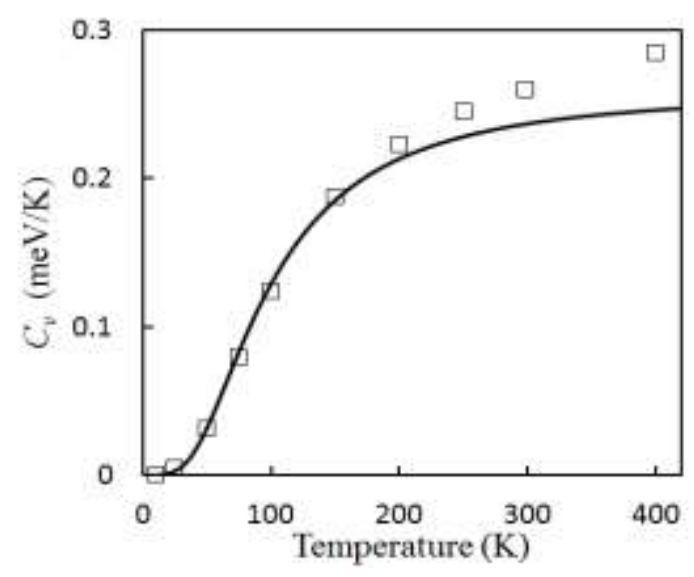

Fig. 6. Specific heat of $\alpha-\mathrm{Fe}$ calculated by computer simulation using the force constants determined using Eq. (2) (solid line) and values observed by specific heat measurement ( $\square$ ). The difference between observed and calculated specific heat above $200 \mathrm{~K}$ is mainly from the contribution of $\alpha-\gamma$ phase transiton of Fe [13].

\section{CONCLUSION}

The correlation effects of atomic thermal displacements in $\alpha$-Fe were estimated from a generalized equation based on the former diffuse scattering analysis. A transformation from correlation effects to force constants was applied. The derived force constants were used to calculate the phonon dispersion relations, phonon density of states, and specific heat of $\alpha-\mathrm{Fe}$ by computer simulation. The calculated phonon dispersion curves and specific heat agree well with the results of inelastic neutron scattering and specific heat measurements, respectively.

\section{ACKNOWLEDGMENT}

One of the authors (Xianglian) wishes to express her thanks for financial support to the Scientific Research Staring Foundation for the Returned Overseas Chinese Scholars, Ministry of Education of China. This research was partially supported by the Ministry of Education, Culture, Sports, Science, and Technology of Japan through Grant-in-Aid for Scientific Research (C), 24510117.

\section{REFERENCES}

1. T. Sakuma, Bull. Electrochem. 11 (1995) 57.

2. T. Sakuma, S. R. Mohapatra, N. Isozaki, et al., J. Non-Cryst. Solids 357 (2011) 559.

3. T. Sakuma, Xianglian, N. Shimizu, et al., Solid State Ionics 192 (2011) 54.

4. Xianglian, S. Siagian, K. Basar, et al., Solid State Ionics 180 (2009) 480.

5. K. Basar, S. Siagian, Xianglian, et al., Nucl. Instrum. Methods Phys. Research A 600 (2009) 237.

6. T. Sakuma, S. R. Mohapatra, H. Uehara, et al., Atom Indonesia 36 (2010) 121.

7. G. Beni and P. M. Platzman, Phys. Rev. B 14 (1976) 1514.

8. W. Böhmer and P. Rabe, J. Phys. C 12 (1979) 2465.

9. O. Kamishima, T. Ishii, H. Maeda, et al., Jpn. J. Appl. Phys. 36 (1997) 247.

10. Makhsun, T. Hashimoto, T. Sakuma, et al., J. Phys. Soc. Jpn. 83 (2014) 074602.

11. V. J. Minkiewicz, G. Shirane, and R. Nathans, Phys. Rev. 162 (1967) 528. 
12. R. Singh, S. Prakash, R. Meyer, et al., Pramana 60 (2003) 547.

13. R.J. Weiss, Solid State Physics for Metallurgists, Elsevier, Amsterdam (2013).

14. Hoshikawa, S. Kakei, H. Yoshinaga, et al., Activity Report on the Utilization of Research Reactors (JRR-3 and JRR-4), JAEA-Review, 100 (2008) 0672008.
15. T. Ishigaki, A. Hoshikawa, M. Yonemura, et al., Nucl. Instrum. Methods Phys. Research A 600 (2009) 189.

16. R. Oishi, M. Yonemura, Y. Nishimaki, et al., Nucl. Instrum. Methods Phys. Research A 600 (2009) 94.

17. S. Koide, Physics ( $3^{\text {rd }}$ ed.), Shokabo, Tokyo, (1997). 